\title{
Bidding Behavior in the Price is Right Game: An Experimental Study
}

\section{Paul Healy and Charles Noussair*}

May, 2003

\begin{abstract}
In this paper, we present an experimental test of a bidding game identical to the one seen on the television game show "The Price is Right". Four players make sequential guesses about the price of an item and the player whose guess is closest to the price without exceeding it wins the item. Patterns of suboptimal behavior in our experimental data are consistent with field data from the actual game show analyzed by Berk, Hughson and Vandezande (1996). Several treatments that simplify the game are used to identify factors that cause subgame perfection to predict poorly.
\end{abstract}

Keywords : Price is Right, Experiment, Bidding, Subgame Perfection

JEL Classification: C7, C9

\section{Introduction}

Subgame perfection is a powerful equilibrium concept that can be very effective in narrowing down the set of Nash equilibria in a dynamic game. Although the logical rationale for subgame perfection is compelling, for outcomes to reflect it, individuals must reason backward in time through the extensive form of the game, and this may be unnatural for many individuals. The descriptive power of subgame perfection is challenging to study because, other than with experimental techniques, it is difficult to find dynamic interactions in which the rules are

Corresponding Author: Noussair: 327 Rich Memorial Building, Department of Economics, Emory University, Atlanta, GA 30322, USA. Email: cnoussa@emory.edu Telephone: (404) 712-8167 Fax: (404) 727-4639.

Healy: M/C 228-77, Division of the Humanities and Social Sciences, California Institute of Technology, Pasadena, CA 91125, USA. Email: pj@ hss.caltech.edu Telephone: (626) 395-4052 Fax: (626) 405-9841.

We thank Tim Cason, John Morgan, and Janet Netz for helpful suggestions. 
explicitly stated and optimal strategies are known and straightforward to calculate. However, Berk, Hughson and Vandezande (1996), hereafter BHV, study a novel data set that has these features as well as very high monetary stakes for players. BHV gather data from a bidding game played on a popular television program, The Price is Right, in which four players bid for a prize that typically has a value of over $\$ 1,000$.

The rules of the bidding game are the following. Four bidders, who are presented with a commonly-used commercially-sold item, each submit a guess of the retail price of the item. The four bidders announce their guesses, which we will refer to as bids in this paper, publicly and in sequence so that the guesses of previous bidders are known at the time a player makes his decision. The player whose bid is the closest to the actual retail price without exceeding the price receives the item and the possibility of winning more prizes later in the program. If all four bids exceed the actual price, the bidding process is repeated. The winner does not make any payment in exchange for receiving the item. ${ }^{1}$ The game has the attractive feature that it is constant-sum: departures from subgame perfection do not occur because there exist gains from cooperation, such as in repeated prisoners' dilemmas (Selten and Stoecker, 1986) or in the centipede game (McKelvey and Palfrey, 1992), nor because of losses from disagreement as in the ultimatum bargaining game (Güth et al., 1982).

In their analysis of the data from The Price is Right, BHV find major discrepancies between the data from the television program and the subgame perfect equilibrium of the bidding game. For example, $\mathbf{t}$ is always optimal for bidder 4 (the fourth and last mover) in the game to either (a) cut off one of the other three bidders by bidding higher than the other bidder by the minimum permissible bid increment, or to (b) bid the minimum possible value. BHV observe that in over $43 \%$ of instances, players do not choose from that simple subset of their strategy set. In the subgame perfect equilibrium, players make bids in descending order; the first player submits the highest bid, the fourth player the lowest. However, in BHV's data, players bid in descending order in only $3.76 \%$ of games. The dichotomy between observed and optimal behavior is striking given the high stakes involved, and the simple rules guiding the best response of player four.

\footnotetext{
${ }^{1}$ The game is of independent interest to economists because it is equivalent to other situations that arise in industrial organization. In one example, a situation in location theory, consider four firms deciding where to locate their businesses (say gasoline stations) on a one-way street or highway. Traffic enters at various points along the street and stops at the first business it encounters. Each of the firms would like to locate in such a way as to maximize the percentage of incoming traffic that reaches it before reaching another firm. Another example, modeled by Cancian et al. (1995), is the scheduling of television evening news by competing networks. This is analogous to the one-way location situation described above in that each network chooses the time to schedule its newscast to maximize the number of viewers for whom its newscast is the first they see after arriving home at the end of the workday.
} 
In this paper, we construct an experiment to explore the stark differences between the theoretical predictions and the data from the game show. ${ }^{2}$ The conclusions reached from our study of this particular game are used to propose conjectures about the types of games in which subgame perfect equilibria might be more likely to be observed. Our analysis of the Price is Right bidding game proceeds as follows. We first replicate properties of the data observed in the game show. We then alter the structure of the game to determine where the failure of subgame perfection arises.

The first avenue we pursue to look for the source of the failure is to consider whether the public nature of decisions causes deviations from subgame perfection. BHV argue that the lack of cutting off behavior is not due to fear of reciprocal cutoffs in response. They cite the fact that cutting other players off early in the game does not lead to an increased likelihood of being cut off in the future. Nevertheless there may exist a social cost to being seen as cutting others off, and it might be viewed as undesirable to be observed doing so. We isolate this effect by running a treatment in which player anonymity is increased, in that other players cannot associate an individual's actions and his identity, and compare behavior and outcomes with the baseline treatment.

BHV argue that bounded rationality is the reason for the departures from optimal behavior. We explore this possibility from three avenues. The first is that we observe the same subjects playing for 50 rounds and check whether decisions improve after experience is acquired. The second avenue we explore is whether the lack of optimal behavior is due to the possibility of resale of the unit in the case that all bids are greater than the actual price. The optimality of cutting off or bidding at the lower bound is dependent on assumptions about future play. If cutoffs induce changes in strategies of other players in the resale round, they may not be optimal to employ. The possibility that there may always be a resale round means that there is no finite

\footnotetext{
${ }^{2}$ Friedman (1998) and Cason and Tenorio (2001) conduct experiments similar in spirit to ours. Friedman's experiment is based on Monty Hall's Three-Door problem on the television game show "Let's make a Deal." He finds significant increases in the incidence of optimal decisions when subjects are required keep track of how their earnings compared with their hypothetical earnings had they pursued alternative strategies. Optimal behavior was also more likely when subjects were shown the results of the decisions of other subjects.

Cason and Tenorio consider another game that occurs in the Price is Right game show. In the game, three players, who move sequentially, each have an opportunity to spin a wheel twice that randomly generates a number from a discrete uniform distribution on $[0,1]$. The numbers generated by the two spins are added to form a score, and the player whose score is closest to but does not exceed 1 wins the game. A player may forego his second spin if he chooses and count the amount of the first spin as his total score. The authors compare behavior in the game show to the behavior observed in a laboratory experiment. They find that behavior is similar in the two settings. In particular, in the two data sets, they observe a similar pattern of suboptimal behavior. The player who moves first tends to decline to make a second spin when
} 
extensive form and therefore it is impossible to reason logically through all of the decision nodes in all possible subgames. We consider this issue by studying data from games with no resale, in which the maximum number of moves for each player is one.

Another reason that decisions may deviate from the optimum is the existence of strategic uncertainty. Decisions early in the game tree are only going to be optimal if early movers believe that future play is likely to be optimal. Subgame perfect equilibria are only likely to be observed if it is common knowledge that future movers will behave rationally. We study this possibility by considering a version of the game with three players and no resale. The existence of fewer players does not simplify the decision of the last mover, but it changes the backward induction problem that must be solved by the first and second movers, who must anticipate the actions of later movers. The next section describes the game and the procedures of the experiment. Section three presents our hypotheses about behavior and outcomes. In section four we present our results, and in section five we provide a summary and some concluding remarks.

\section{Procedures}

The experiment consisted of nine sessions. All of the sessions were conducted in classrooms or computer labs in the Krannert School of Management at Purdue University, and the subjects participating in this experiment consisted entirely of undergraduate students from Purdue University. In each session, subjects played the Price is Right bidding game 50 times. We will refer to each play of the game as a period. The winner of each period was awarded $\$ 2$ for a maximum total of $\$ 100$ payment given to each group of four subjects. Sessions took on average approximately two hours.

\subsection{The Public Treatments}

Sessions 1-4, which constituted the PR4, (Public/Resale/4Bidder) treatment, were conducted by hand. In each of these four sessions, there were four subjects, who interacted with each other for 50 periods. Participants were seated at desks facing the chalkboard. The instructions for the experiment were read aloud as participants followed along with printed copies. The instructions were available for reference for the duration of the experiment. Subjects were then given a chance to ask questions regarding the procedure of the experiment. Following the instructions, one practice period of the experiment was conducted which did not count for money. After the practice period, fifty periods were conducted with monetary payments. The

the probabilty that the sum of the two spins exceeds one is high, even when it is optimal for the player to 
data were recorded both on paper and on the chalkboard. At any time, subjects could observe the history of all subjects' actions from between ten and twenty immediately preceding periods.

The timing of activity in each individual period was as follows. After fifteen seconds, the experimenter signaled to bidder 1 that he could declare an integer between 1 and 1000 (inclusive) whenever he was ready. The fifteen-second interval was meant to encourage subjects to take time to analyze the decision situation they faced. After bidder 1 verbally submitted his number, it was recorded on the chalkboard so that all other subjects could observe the choice. After another fifteen-second delay, bidder 2 was allowed to submit her number. Again, the number was recorded on the chalkboard. This same process continued with bidder 3 and bidder 4 . After bidder 4's number was recorded on the chalkboard, the experimenter rolled a 10-sided die and a 100-sided die simultaneously. The ten-sided die had values 0 through 9 and the 100 -sided die had values 0 through 99. The two numbers were concatenated to form a three-digit number with the 10-sided die representing the first digit and the 100-sided die representing the last two. If both dice returned zero, the experimenter's number was 1000 . The subjects were occasionally given the chance to roll the dice and to read the numbers.

After the experimenter's number was recorded on the chalkboard, if the experimenter's number was greater than at least one player's number, the winner for the period was selected. The winner was the bidder whose choice was closest to the experimenter's number without exceeding it. The winner's bid was circled, he was informed that he had won $\$ 2$, and the experiment proceeded to the next period. A second experimenter seated at the back of the room recorded on paper all of the data from the chalkboard.

In sessions 1-4, if all four bidders' numbers were greater than the experimenter's number, then no winner was determined, and play continued with a resale round. The procedure for resale rounds was identical to the initial round of each period. When described to the subjects, a resale round was labeled in a way that underscored the link between the original round and the resale round. For example Period 3.1 was the first resale round in Period 3. If all bidders' numbers were again greater than the experimenter's number in Period 3.1, then play continued to Period 3.2, etc. Subjects were frequently reminded that no money would be awarded if all four numbers were greater than the experimenter's number and that resale rounds would continue until a winner was determined for the period. Subjects were also reminded that 50 periods would be played during the session (not including the practice period at the beginning), so it was common knowledge that the $\$ 2$ prize would be awarded exactly 50 times.

spin an second time. 
In sessions 1 and 2, subjects rotated positions after 25 periods in a pattern where the subject initially acting as bidder 4 and the subject initially acting as bidder 1 switched roles, as did those subjects acting as bidders 2 and 3. In sessions $3-4$ and $6-9$, subjects rotated once every 10 periods, so that the subject initially acting as bidder 1 became bidder 4 after 10 periods, bidder 3 after the $20^{\text {th }}$ period, bidder 2 after the $30^{\text {th }}$ period, and finally bidder 1 for the final 10 periods. All other subjects were rotated in the same order of "1-4-3-2-1", that is 10 periods in the role of player 1 was followed by 10 periods in the role of player 4 , followed by 3 , etc. This rotation scheme encouraged backward induction since the subject acting as bidder 3 had previously been acting as bidder 4 and thus understood bidder 4 's incentive to cut off certain bids. The rotation scheme also allowed a subject to rotate from the "powerless" bidder 1 position to the "powerful" bidder 4 position and reciprocate the behavior of the other three players.

Sessions 6 and 7, which are referred to as the PN4 treatment (Public/NoResale/4bidder), differed from 1-4 only in that there was no resale round. There was exactly one round played for 50 periods. This meant that there could be less than 50 prizes sold over the course of the session. Sessions 8 and 9 were identical to the PN4 sessions except for the fact that there were three bidders rather than four, and are designated as the PN3 treatment.

\subsection{The Anonymity Treatment AR4}

Session 5, which constituted the AR4 (Anonymity/Resale/4bidder) treatment, was computerized and consisted of three groups interacting simultaneously in a large computer lab. These three sessions increased the level of anonymity in the experiment by ensuring that subjects could not associate the actions and the identity of other players. Under the AR4 treatment, the 12 subjects were spaced throughout the lab and instructed to direct their Web browser to the experiment's web site. Each subject was given a unique login ID and password. The players received a game name and a starting player number. They then reviewed instructions explaining the bidding procedure and the use of the Web page for viewing and submitting bids. Subjects

were given printed copies of these instructions to which they could refer throughout the experiment.

We conducted fifty periods, not including one practice period that did not count toward subjects' earnings. Each round consisted of the following sequence of events: After logging into their assigned game, each subject continually viewed a table which displayed the round number, each of the bidders' bids as they were made, the experimenter's number, and the winning number for each of the previous rounds, including the current round. At the bottom of the table was a 
status bar that indicated how much money the subject had won during the course of the experiment.

At the beginning of the round, bidder 1's browser displayed a pop-up window into which he was able to input a number after 15 seconds. After entering the number, all players saw it appear in their table under "Player 1" for the current round. Bidder 2's browser then displayed a pop-up window after a 15-second delay into which she could enter a number. Again, the number was immediately made available to all other players in the game. The process continued with bidders 3 and 4.

After bidder 4 entered her number, the computer randomly generated a number from a discrete uniform distribution between 1 and 1000 and determined the winner. The experimenter's number and the winning player's number were highlighted in all of the players' tables. The game was then advanced to the next period. If all players entered numbers greater than the experimenter's number, then the next round was a resale round (labeled for example 3.1 for the first resale round of period 3) and a " 0 " was placed in the column of the table labeled "Winner". The resale round process continued until a winner for the period was determined.

After 10 periods, the Web browser instructed subjects to log out of their game and log into the next "rotation" of 10 periods. The bidding procedure was identical, but the subjects were rotated to a new bidder position according to the aforementioned "1-4-3-2-1" scheme. Subjects were not informed of the rotation scheme and were thus unable to identify positively any other subject's role as she rotated through the bidder positions. Therefore, it was difficult for a subject to target another subject for reciprocation. In order to punish a player by cutting her off, one risked a $2 / 3$ probability of cutting off the wrong player. ${ }^{3}$ After the $10^{\text {th }}$ period of the $5^{\text {th }}$ rotation (the $50^{\text {th }}$ period in total), the subjects were told to sit quietly until all players were finished, so that the last group of four subjects to finish could not identify the other members of their group.

\section{Hypotheses}

\section{1: Hypotheses derived from Game Theory}

BHV derived several testable game-theoretic propositions about behavior in the game. They require varying degrees of rationality, and we generally interpret more support for them in

\footnotetext{
${ }^{3}$ In principle, a subject could calculate the current position of a player who cut her off earlier under the assumption that all bidders followed the same rotation. However, there was no way that she could be certain that all players rotated in the same pattern as she did. Furthermore, it would take at least three rotations before players could reasonably conjecture the entire rotation scheme. We used the 1-4-3-2-1 rotation scheme in the Anonymity treatment because it facilitated a direct comparison with the data from sessions 3 and 4, which used the same rotation scheme.
} 
different treatments as evidence of a greater degree of rational behavior in that treatment. Hypotheses 1a-c concern the strategies chosen in the experiment.

Hypothesis 1a: Local Best Response Strategies: The last bidder cuts off one of the previous bidders or bids 1 .

Hypothesis 1b: Optimal last mover behavior - The last bidder chooses the element from the set of four actions listed in hypothesis la that maximizes his probability of winning.

Hypothesis 1c: Subgame Perfect ordering - Players bid in descending order.

Hypothesis 1a requires that the last bidder choose a strategy from a set of "local" best response strategies, where the local best responses yield a strictly higher payoff than any other strategy in some interval above the local best response. It can be easily shown that adopting any strategy that does not involve cutting off a previous bidder or bidding 1 is not a best response strategy. For example, if the first three bidders submit bids of 100,600, and 750, the four actions of bidder four that are local best responses consistent with hypothesis 1a are 101, 601, 751 and 1. Every other strategy yields a strictly lower expected payoff than the largest local best response below the given strategy. For example, a strategy of submitting 200 gives a lower expected payoff than submitting 101. By submitting 200, bidder 4 wins at any price between 200 and 600, a $40.1 \%$ chance of winning in the current round in addition to a probability of winning in a future round if the item has to be resold. By submitting 101, bidder 4 wins at any price between 101 and 600 , a $50 \%$ chance of winning in the current round in addition to the probability of winning by resale.

Each of the four local best response strategies consistent with hypothesis 1a leads to a certain probability of winning. By picking the lowest number in the appropriate interval, the fourth bidder maximizes his probability of winning. Hypothesis $1 \mathrm{~b}$ is that the fourth bidder chooses the one local best response strategy that gives him the highest expected return for the entire period. In the example in the last paragraph, the probabilities of winning for the four actions are (a) for 101, .5 +.1(prob. of winning on resale), (b) for $601, .2+.1$ (prob. of winning on resale), (c) for $751, .25+.1$ (prob. of winning on resale), and (d) for $1, .1$. Thus the option with the highest expected payoff for bidder 4 would be to bid 101, and hypothesis $1 \mathrm{~b}$ predicts that 101 would be chosen in the example. ${ }^{4}$

\footnotetext{
${ }^{4}$ In their analysis, BHV make the assumption that bidders will choose the same strategies in resale rounds as they did in the initial round; the probability of any given bidder winning the period can be found by the formula $p($ Win $\$ 2)=\frac{p(\text { WinCurrentRound })}{1-p(\operatorname{Re} \text { saleRoundQcurs })}$. To see that this is true, consider that if $a$ is the probability that
} 
Hypothesis 1c is a property of the subgame perfect equilibrium of the game. See BHV for a derivation. For the PR4 and AR4 treatments, in which the distribution of prices is drawn from a discrete uniform distribution on the interval [1,1000], the actions chosen along the subgame perfect equilibrium path are bidder 1 bids 779, bidder 2 bids 557, bidder 3 bids 334 and bidder 4 bids 1. In PN4, the equilibrium bids of the four players are 751, 501, 251 and 1. In PN3, they are 668,334 , and 1.

Subgame perfection requires strong assumptions about players' beliefs. In particular, it requires common knowledge that later movers in the game are rational. However, weaker assumptions about the beliefs of the four subjects place some restrictions on outcomes. Suppose that each subject has "rational expectations" in the sense that he knows (a) the distribution of the true price of the item, and (b) his probability of winning the prize given his position in the bidding order. BHV show that under these two assumptions the outcomes must have the properties described in hypotheses $2 \mathrm{a}$ and $2 \mathrm{~b}$ below.

Support for hypotheses $2 \mathrm{a}$ and $2 \mathrm{~b}$ is considered as a failure to reject assumptions (a) and (b) concerning rational expectations. The methodology of the experiment includes informing the subjects about the distribution of experimenter prices and using physical devices (dice) to convince the subjects that they indeed face a uniform distribution. This represents an attempt to impose assumption (a). Assumption (b), that the subjects correctly anticipate their probability of winning, is presumably more and more likely to be satisfied as subjects repeat the process over the course of their session.

Hypothesis $2 \mathrm{c}$ requires that the winning probabilities be those that occur in the subgame perfect equilibrium. BHV derive the results for the case of 4 bidders and resale, but it is straightforward to show that analogous results hold in the absence of resale.

Hypothesis 2a: Rational expectations payoff ranking - The last bidder wins with at least as high a probability as the next-to-last bidder. The next-to-last bidder wins with at least as high a probability as any previous bidder.

Hypothesis 2b: RE payoff lower bounds - If there are four bidders, and resale is possible, the fourth bidder wins with a probability of at least 1/3. If there are four bidders, and resale is not possible, the fourth bidder wins with a probability of at least 1/4. If there are three bidders, and resale is not possible, the third bidder wins with a probability of at least 1/3.

Player 4 wins the current round and $b$ is the probability that nobody wins the current round, thus forcing a resale round, and if all players chose the same bids on all subsequent resale rounds, then the probability of winning the $\$ 2$ is the infinite series $\quad a+b\left(a+b\left(a+\ldots=a \frac{1}{1-b}\right.\right.$ 
Hypothesis 2c: Subgame Perfect Ordering - If there are four bidders and resale is possible, the first three bidders win with probability 2/9 and the fourth bidder wins with probability 1/3. If there are four bidders and no resale is possible, each bidder wins with probability 1/4. With three bidders and no resale, each bidder wins with probability 1/3.

\subsection{Bounded rationality}

We explore several possibilities about the source of the departures from subgame perfection that BHV identify. They are listed below as hypothesis $3 \mathrm{a}-3 \mathrm{~d}$. Hypothesis $3 \mathrm{a}$, that decisions accord more with the predictions of rational models after repetition of the game, focuses on a pattern that is typical of many experimental studies, particularly those involving interactions that are complex. Although most contestants on the TV game show that BHV analyze presumably have considerable experience watching the show before participating, their experience consists of observation rather than prior participation. In the experiment we are able to track behavior of the same individuals over 50 rounds and observe any changes over time. If hypothesis $3 \mathrm{a}$ is supported, it would suggest that sufficient experience would reduce or eliminate the anomalous behavior.

Hypothesis $3 \mathrm{~b}$ notes that behavior is more consistent with the subgame perfect equilibrium under the AR4 treatment than under PR4. As we suggested in the introduction, players may not wish to be seen cutting off others. If this is the case, the anonymity provided in the AR4 treatment would lead to more cutoff behavior. The anticipation of cutoff behavior would then lead to behavior more consistent with the subgame perfect equilibrium on the part of earlier movers. The only difference between the two treatments (other than the computerized interface in AR4) is the inability to associate players' decisions and identity.

Hypothesis $3 \mathrm{c}$ claims that removing the ability to resell would lead to behavior more in accordance with the subgame perfect equilibrium. The absence of resale ensures that each player only has one decision node, which simplifies the game. If resale is possible, each player's strategy must specify their action at each possible decision node in any number of potential resale rounds, and players must form beliefs about how others will bid in the resale round. Because of the existence of so many additional subgames that are introduced with resale, the computational burden is eased considerably if no resale is possible. The hypothesis is evaluated by comparing the data from PR4 and PN4. Hypothesis 3d has a similar spirit and suggests that decisions would be more consistent with subgame perfection in a three-player version of the game with no resale. This type of simplification would further ease the computational burden for players other than the last mover, although there is no reason to suppose that it would directly simplify the decision of 
the last mover. Hypothesis $3 \mathrm{~d}$ therefore applies with particular force to hypotheses $1 \mathrm{c}, 2 \mathrm{~b}$ and $2 \mathrm{c}$, which depend on behavior of players other than the last mover, asserting that those hypotheses are more likely to be supported under PN3 than under PN4.

Hypothesis 3a: Support for hypotheses 1 and 2 increases with repetition of the game. Hypothesis 3b: Support for hypotheses 1 and 2 increases when decisions are anonymous. Hypothesis 3c: Support for hypotheses 1 and 2 increases when there is no resale of the item. Hypothesis 3d: Support for hypotheses 1 and 2 increases when there are fewer players.

\subsection{Reciprocity}

Hypothesis four concerns the incidence of cutoff behavior and its dependence on the number of cutoffs experienced and observed. Hypothesis $4 \mathrm{a}$ asserts the existence of reciprocal cutoffs. According to the hypothesis, subject $i$ is more likely to cut off another subject $j$ the more often $j$ has cut him off previously. This is consistent with the exercise of revenge for earlier cutoffs. Hypotheses $4 \mathrm{~b}$ and $4 \mathrm{c}$ concern the presence and nature of the learning process on the part of agents and the way subjects learn cutoff behavior. Hypothesis $4 \mathrm{~b}$ asserts the existence of behavior that is consistent with learning about the optimality of cutting off from the experience of being cut off. The hypothesis claims that subject $i$, after he has been cut off by subject $j$, will be more likely to cut off subjects other than $j$. Hypothesis $4 \mathrm{c}$ asserts that subject $i$ is more likely to cutoff subject $j$ the more total cutoffs he observes of players other than himself, therefore learning to cut off by observing cutoff behavior.

Hypothesis 4a: If subject $j$ cuts off subject $i$, the likelihood that i cuts off $j$ increases.

Hypothesis $4 b$ : If subject $i$ is cut off by subject $j$, the likelihood that $i$ cuts off subject $k$ increases.

Hypothesis 4c: If subject $k$ is cut off by subject $j$, the likelihood that $i$ cuts off other subjects increases.

\section{Results}

\subsection{Tests of Game Theoretic Predictions and Comparison to BHV}

Table 1 indicates the percentage of bids that were consistent with hypotheses $1 \mathrm{a}$ and $1 \mathrm{~b}$ in each treatment. The last player's bid is a local best response in the sense of hypothesis 1a if it cuts 
off a previous bid or is equal to 1 . The bid is optimal in the sense of hypothesis $1 \mathrm{~b}$ if it maximizes the probability of winning, taking into account the possibility of resale where appropriate. The data in the third column are the percentage of choices that are "near" a local best response strategy, defined as less than 50 above another bidder's choice or between 1 and 50 . These data account for bids that satisfy hypothesis 1 a if a margin of 50 is allowed.

\section{[Table 1: About Here]}

The data in table 1 for the PR4 treatment indicate that the incidence of use of local best response strategies is at about the same level as in the data of BHV. Bidder 4 cuts off another bidder or bids 1 for $63.6 \%$ of the time compared to $56.5 \%$ of the time in the BHV study. In the first ten periods of our PR4 treatment, the percentage is 53.3\%. However, we obtain considerably stronger support for hypothesis 1a if we classify choices less than 50 currency units above a local best response action as consistent with the hypothesis. The third column comprises at most $20 \%$ of the possible range of bids but accounts for $79 \%$ of the total observations in PR4. The proportion of decisions that are exactly optimal is $46.3 \%$.

The incidence of local best response and optimal decisions is higher under the Anonymous treatment. The incidence of decisions that are optimal increases from $46.3 \%$ to $57.2 \%$ under anonymity, and larger increases occur for the two measures of consistency with hypothesis 1a. A pooled variance $t$-test rejects the hypothesis that the incidence of local best response behavior is equal in the two treatments at $p<.05(t=2.17)$. We also reject the hypothesis that the incidence of optimal behavior is equal at $p<.05(t=1.97)$. For the percentage of bids that are optimal in the sense of hypothesis 1a while allowing a positive deviation of 50 , we cannot reject the hypothesis of equality between the two treatments at the $p=.05$ level $(t=$ 1.46). Anonymity appears to encourage some players to cut off others when they would bid up to 50 higher than a cutoff strategy if their decisions were made public.

Removing the possibility of resale induces an increase of similar magnitude in the incidence of optimal and local best response behavior as the introduction of anonymity. The hypothesis that the incidence of local best response behavior is identical between PR4 and PN4 can be rejected at the $p<.05$ level $(t=2.03)$ and the analogous hypothesis can be rejected for optimal behavior $(t=1.89)$. However, when an interval of 50 is allowed as a positive deviation from a local best response strategy, the hypothesis of equality cannot be rejected at conventional significance levels $(t=1.18)$. The hypotheses of equality of the three measures between PN4 and PN3 cannot be rejected at the 5\% level. However, the table shows that for the last 10 periods of 
PN3, all decisions the last mover takes are local best responses and $80 \%$ are optimal. Thus, removing the resale property, reducing the number of agents to three, and giving players sufficient experience induces highly optimal decisions and essentially eliminates the anomalous behavior.

Although experience seems to improve decisions in the PN3 treatment, experience does not appear to have a positive effect overall. While decisions in PR4 and PN3 are better than average in the last ten periods, those in AR4 and PN4 are not. The dynamics whereby decisions of the last mover change over time are explored later in section 4.2 below.

\section{[Table 2: About Here]}

Table 2 shows the winning percentage by bidder and allows us to consider the level of support for hypothesis two. The table indicates considerable support for Hypotheses $2 \mathrm{a}$ and $2 \mathrm{~b}$, but not for $2 \mathrm{c}$. The last mover wins more often than any of the other players in all treatments. The next to-last mover wins more often than the earlier movers in all cases but one (in PR4, player 1 wins very slightly more often than player 3 ). The frequencies of the last mover winning are consistent with hypothesis $2 \mathrm{~b}$ in all cases. However, hypothesis $2 \mathrm{c}$, which stipulates win frequencies consistent with the subgame perfect equilibrium, is not supported. Late movers tend to take advantage of non-equilibrium play of earlier movers and win with greater probability than under the subgame perfect equilibrium.

Tables $3 \mathrm{a}$ and $3 \mathrm{~b}$ show the frequency of each possible ordering of the magnitude of bids in a single round, from greatest to least. Table 3 a contains the pooled data from PR4 and AR4 as well as from PN4, while $3 \mathrm{~b}$ contains the data from PN3. The subgame perfect equilibrium ordering is 1234 (for PN3 it is 123). Bidders bid in descending order. The data in the table reveal that hypothesis $1 \mathrm{c}$ is firmly rejected in the data from four player games with resale, as it is in the empirical analysis of BHV. Bidders rarely bid in descending order, as they would in the subgame perfect equilibrium. The frequencies are close to those reported by BHV. Only $1.69 \%$ of the time is the bidding order the one predicted in the subgame perfect equilibrium. In contrast, if bidding were completely random, the bids would be in descending order $4.2 \%$ of the time. In our data, as well as the data of BHV, each of the 24 possible orderings occurs in at least $1 \%$ of the rounds, representing a remarkable degree of variability in outcomes. Similar patterns are obtained in PN4. However, in PN3 the three bids are in declining order in $29 \%$ and bidder three is the lowest bidder in 54\% of all periods, considerably greater than if bidding were random (16.7\% would be in declining order and $33.3 \%$ of the time, the last mover would be the low bidder). In the last 10 
periods $40 \%$ of plays result in the predicted ordering, and player 3 is the low bidder in $70 \%$ of periods. This is further indication that the PN3 treatment induces decisions closer to equilibrium behavior.

[Table 3: About Here]

\subsection{Bounded Rationality and Reciprocity}

We consider here the dynamics over time in cutoff behavior. In particular we study the effect of several plausible factors that can account for changes in the incidence of cutoff behavior over time. We estimate a logit model in which the dependent variable $c_{i j}{ }^{t s}$ takes on a value of 1 if player $i$ cuts off player $j$ in round $s$ of period $t$. $c_{i j}{ }^{t s}$ equals zero if $i$ fails to cut off $j$. Only the observations where it is optimal for $i$ to cut off $j$ and where $i$ is the last mover in the round are included in the estimation. The first influence on cutoff behavior that we consider is pure experience with the decision situation. A positive coefficient on Period, which takes on a value from 1 to 50 equal to the current period number, would indicate that there is a trend over time that other variables cannot explain and would suggest an increase in cutoff behavior related purely to deductive reasoning independent of the number of cutoffs observed or experienced. The second variable is Anonymous, which takes on a value of 1 if the data are from the AR4 treatment. The third variable, CutoffsObserved, equals the number of times that any player cuts off a player $k \neq i$. A positive coefficient on CutoffsObserved would indicate that subjects who observe more cutoffs are more likely to cut off others later. This may occur if observation of the cutoff strategy makes players recognize its optimality or increase their willingness to employ it.

The variable CutoffbyOthers indicates the number of times that $i$ has himself been cut off by a player $k \neq j$. If the coefficient of this variable is positive, there an additional tendency for players to respond to being cut off by adopting cutoff behavior themselves beyond the effect of CutoffsObserved. It would suggest that experiencing a cutoff is an especially powerful way to illustrate its optimality. The variable CutoffByJ is the number of times that $i$ has been cut off by $j$. Positive significance of the coefficient of this variable would indicate the presence of reciprocity, since it would reveal that players are especially likely to target players who cut them off for reciprocation. The data reported in table 4 are logit estimates with dependent variable $c_{i j}{ }^{t s}$ and the independent variables given above. ${ }^{5}$ Only the observations for which it is optimal for $i$ to cut off $j$ are included.

\footnotetext{
${ }^{5}$ CutoffbyOthers $=\#$ of times the subject currently acting as bidder 4 has been cut off between periods 1 and $t-1$ (inclusive) by players other than $j$.
} 
[Table 4: About Here]

BHV find in their data that players were more likely to adopt a local best response strategy if they had observed one previously, but there was no additional effect from being themselves the victims of a cutoff. That is, learning the optimality of cutoff behavior from observation was as effective as from experience. BHV also found no evidence of reciprocal cutting off behavior. Cutting off a player did not appear to lead to future revenge because the player was no likelier to be cut off in subsequent rounds than if she had not cut off another player. The estimates we obtain with the data from 4 players and resale, also shown in table 4 , lead to the same conclusions. The variable CutoffsObserved is positive and significant indicating that subjects cut off others more often, the more cutoffs they observe. BHV interpret this effect as indicating that subjects are not aware beforehand of the optimality of the strategy and learn it from experience. We believe that it is a more plausible explanation in that it is a priori not common knowledge that reciprocation does not occur for cutoffs and subjects learn this as they repeat the game.

The variable CutoffbyOthers is insignificant. This indicates that there is no marginal increase in cutoff behavior from a player who has been cut off. This is also in agreement with the findings of $\mathrm{BHV}$ that there is no additional cutoff behavior induced by an experience of being personally cut off. The variable Cutoffby $J$ is not significant, indicating that there is no tendency toward direct reciprocity in the data. These effects are all consistent with BHV although somewhat at odds with other experimental research (see for example Fehr et al. (1993) or Fehr and Gächter (2000)) in which reciprocation is a prominent feature of the data. The coefficient on Period is significantly negative, indicating that if no cutoff behavior occurs in a period, future cutoff behavior is less likely. Anonymous is insignificant, suggesting that the presence of anonymity itself did not increase the probability of a cutoff, holding constant the previous history of cutoff behavior in the session.

The results from the estimation are different when no resale occurs. The three-player treatment PN3 is of particular interest because of the results reported previously suggesting

Cutoffs Observed $=$ Total \# of times that players other than $i$ have been cut off in the session between periods 1 and $t-1$ (inclusive).

Period $=$ \# of periods that have already elapsed in the current session.

CutoffByJ = Total \# of times that subject $j$ cut off subject $i$ before the current period.

CutoffByOthers $=$ Total \# of times that subjects other than $j$ cut off subject $i$ before the current period.

Anonymous $=1$ if Anonymity treatment is in effect. 
behavior was relatively close to equilibrium behavior. We interpret the estimates as reflecting a better understanding of the game. CutoffsObserved is insignificant, which indicates that players were not made aware of the optimality of the cutoff strategy by observing it and suggests that they were already aware of it from introspection. Unlike PR4, none of the variables is significant. This lack of significance is consistent with rational behavior. CutoffbyOthers is negative and CutoffbyJ is positive in sign, although neither is significant at the 5\% level. Thus there is may be a slight tendency toward reciprocal behavior. A positive Cutoffby $J$ indicates that player $i$ is more likely to cut off another player who cut him off earlier, and a negative CutoffbyOthers means that $i$ reacts to a cutoff from $k$ by being less likely to cut off $j$, positively reciprocating not being cutoff by $j$. Thus it appears that the Price is Right bidding game with four players and resale is too complex for the behavioral principles typically observed in the laboratory to manifest themselves. However, the relatively simple game in PN3 generates data that is highly consistent with rational behavior.

\section{Discussion}

In this study, we replicate many empirical patterns that BHV identify in their data from the Price is Right television show bidding game. For example, we find that at the beginning of our sessions, the incidence of cutoff behavior is roughly the same as in the BHV study. The probability of a player winning the prize given his position in the bidding order is very close in the two studies. We also reproduce the very diffuse results BHV obtain on the order of bids. The data from our experiment demonstrate the extent to which the laboratory can attain parallel outcomes with corresponding field data, even though the stakes on the game show are several hundred times greater than the two dollar prizes in our experiment and the game show is conducted in a natural setting.

In our PR4 data, which corresponds to the game played on the television show, the subgame perfect equilibrium was not an accurate predictor of the actual bids. However, we show that reducing the number of players to three, eliminating the possibility of resale of the item, and giving participants some practice with the game induces decisions that are close to optimal ones. The deviations from equilibrium play in PN3 for which there is any evidence at all appear to be mostly due to reciprocal cutoffs and accord with previous experimental results. Thus the anomalous behavior in the bidding game appears to be due to resale, as well as the relatively large number of players. When these properties are removed, the game generally behaves in a manner consistent with standard theory. Behavior in PN3 cannot be classified as "anomalous". 
We believe that the treatments PR4 and PN3 behave differently for two reasons. The first is that the existence of resale introduces additional subgames that are off the equilibrium path and that are reached when all players bid higher than the experimenter price. Their existence increases the number of contingencies that a player must analyze at each of his decision nodes. Although they are not reached in equilibrium, these subgames are likely to be reached in the early periods when subjects are becoming familiar with the decision context. The fact that these subgames are reached complicates the calculation of expected payoffs for different actions at earlier decision nodes. The realization of relatively high payoffs out of equilibrium can result in reinforcement of actions (see for example Erev and Roth, 1998; or Camerer and Ho, 1999) that lie off of the equilibrium path and make their use persistent. We conjecture that more generally, subgame perfection will predict better when there are fewer out of equilibrium outcomes, particularly those that result in relatively high payoffs for one or more players.

A three-player version of the game simplifies the decisions of early movers in the game because it is more likely that they have confidence that all later movers will induct backwards. This makes it easier to create the common knowledge of rationality that subgame perfection requires. However, it also improves the decisions of the last mover, whose decisions were closer to optimality under PN3 than under PN4. This appears to occur because decisions of all three players stabilize more quickly, meaning there are relatively few actions of earlier bidders to which the last mover must respond. Repeated experience with similar actions of previous movers facilitates the calculation of optimal best response to those actions and thus the creation of common knowledge of rationality on the part of earlier players. The complexity of the four-player game with resale prevents this from occurring because decisions are highly variable from period to period. It may be the case that in general, games with fewer players are more likely to converge to and achieve subgame perfect outcomes because of the relative ease of making it common knowledge that players are rational. 


\section{References}

Berk, J., Hughson, E., Vandezande, T., 1996. The price is right but are the bids? An investigation of rational decision theory. American Economic Review 86, 954-970.

Camerer, C., Ho, T., 1999. Experience-weighted attraction learning in normal form games.

Econometrica 67, 827-874.

Cancian, M., Bills, A., Bergstrom, T., 1995. Hotelling location problems with directional constraints: an application to television news scheduling. Journal of Industrial Economics 43, 121-124.

Cason, T., Tenorio, R., 2001. To spin or not to spin? Natural and laboratory experiments from The Price is Right. Economic Journal 112, 170-195.

Erev, I., Roth, A.E., 1998. Predicting how people play games: reinforcement learning in games with unique mixed strategy equilibria. American Economic Review 88, 848-881.

Fehr, E., Gächter, S., 2000. Fairness and retaliation: the economics of reciprocity. Journal of Economic Perspectives 14(3), 159-181.

Fehr, E., Kirchsteiger, G., Riedl, A., 1993. Does fairness prevent market clearing? An experimental investigation. Quarterly Journal of Economics 108, 437-459.

Friedman, D., 1998. Monty Hall's three doors: construction and deconstruction of a choice anomaly. American Economic Review 88, 933-946.

Guth, W., Schmittberger, R., Schwarze, B., 1982. An experimental analysis of ultimatum bargaining. Journal of Economic Behavior and Organization 3, 367-388.

McKelvey, R., Palfrey, T., 1992. An experimental study of the centipede game. Econometrica 60, 803-836. 
Selten, R., Stoecker, R., 1986. End behavior in sequences of finite prisoners' dilemma supergames: a learning theory approach. Journal of Economic Behavior and Organization 7, 4770. 
Table 1: Percentage of Bids Consistent with Hypotheses 1a and 1b, All Treatments

\begin{tabular}{|l|l|l|l|}
\hline Session & $\begin{array}{l}\text { Percent of bids that are } \\
\text { local best responses }\end{array}$ & $\begin{array}{l}\text { Percent of bids that are } \\
\text { less than 50 greater than } \\
\text { a local best response bid }\end{array}$ & $\begin{array}{l}\text { Percent of bids that are } \\
\text { optimal }\end{array}$ \\
\hline $\begin{array}{l}\text { PR4/ All } \\
\text { Data }\end{array}$ & $63.6 \%$ & $79.0 \%$ & $46.3 \%$ \\
\hline $\begin{array}{l}\text { PR4/Last 10 } \\
\text { Periods }\end{array}$ & $72.9 \%$ & $81.3 \%$ & $43.8 \%$ \\
\hline $\begin{array}{l}\text { AR4/ All } \\
\text { Data }\end{array}$ & $80.3 \%$ & $92.4 \%$ & $57.16 \%$ \\
\hline $\begin{array}{l}\text { AR4/Last } 10 \\
\text { Periods }\end{array}$ & $74.4 \%$ & $94.9 \%$ & $61.5 \%$ \\
\hline $\begin{array}{l}\text { PN4/ All } \\
\text { Data }\end{array}$ & $81.0 \%$ & $91.0 \%$ & $58.0 \%$ \\
\hline $\begin{array}{l}\text { PN4/Last 10 } \\
\text { Periods }\end{array}$ & $60 \%$ & $90 \%$ & $40 \%$ \\
\hline $\begin{array}{l}\text { PN3/ All } \\
\text { Data }\end{array}$ & $76.0 \%$ & $93.0 \%$ & $61.0 \%$ \\
\hline $\begin{array}{l}\text { PN3/Last } 10 \\
\text { Periods }\end{array}$ & $100 \%$ & $100 \%$ & $80 \%$ \\
\hline \begin{tabular}{l} 
Berk et al \\
\hline
\end{tabular} & $56.5 \%$ & N/A & N/A \\
\hline
\end{tabular}


Table 2: Winning Percentages by Player, All Treatments

\begin{tabular}{|l|l|l|l|l|}
\hline Session & $1^{\text {sl }}$ Player & $2^{\text {nd }}$ Player & $3^{\text {rd }}$ Player & $4^{\text {th }}$ Player \\
\hline PR4/ All Data & 20.2 & 17.5 & 20.0 & 42.4 \\
\hline $\begin{array}{l}\text { PR4 } \\
\text { Predicted }\end{array}$ & 22.2 & 22.2 & 22.2 & 33.3 \\
\hline $\begin{array}{l}\text { AR4/ All } \\
\text { Data }\end{array}$ & 13.2 & 21.1 & 21.6 & 41.2 \\
\hline $\begin{array}{l}\text { AR4 } \\
\text { Predicted }\end{array}$ & 22.2 & 22.2 & 22.2 & 33.3 \\
\hline $\begin{array}{l}\text { PN4/ All Data } \\
\text { PN4 }\end{array}$ & 10.7 & 11.9 & 28.6 & 48.8 \\
\hline Predicted & 25 & 25 & 25 & 25 \\
\hline $\begin{array}{l}\text { PN3/ All Data } \\
\text { PN3 }\end{array}$ & 33.6 & 26.1 & 54.3 & ----- \\
\hline Predicted & 33.3 & 33.4 & ----- \\
\hline
\end{tabular}


Table 3a: Bidding Order Percentages, 4-Player Data, All Sessions

\begin{tabular}{|c|c|c|r|}
\hline $\begin{array}{c}\text { Bidding Order } \\
\text { (Descending) }\end{array}$ & $\begin{array}{c}\text { Percentage of Periods } \\
\text { Sequence is } \\
\text { Observed: } \\
\text { Pooled Data from PR4 } \\
\text { and AR4 }\end{array}$ & $\begin{array}{c}\text { Percentage of } \\
\text { Periods Sequence } \\
\text { is Observed: BHV } \\
\text { Data }\end{array}$ & $\begin{array}{c}\text { Percentage of } \\
\text { Periods } \\
\text { Sequence is } \\
\text { Observed: PN4 }\end{array}$ \\
\hline $1234^{\star * *}$ & $1.69 \%$ & $3.76 \%$ & $6.0 \%$ \\
\hline 1243 & $7.00 \%$ & $2.42 \%$ & $8.0 \%$ \\
\hline 1324 & $2.42 \%$ & $4.84 \%$ & $3.0 \%$ \\
\hline 1342 & $4.35 \%$ & $2.69 \%$ & $3.0 \%$ \\
\hline 1423 & $5.31 \%$ & $3.76 \%$ & $6.0 \%$ \\
\hline 1432 & $9.90 \%$ & $3.23 \%$ & $1.0 \%$ \\
\hline 2134 & $3.14 \%$ & $2.96 \%$ & $5.0 \%$ \\
\hline 2143 & $6.04 \%$ & $4.30 \%$ & $1.0 \%$ \\
\hline 2314 & $1.45 \%$ & $2.69 \%$ & $5.0 \%$ \\
\hline 2341 & $1.45 \%$ & $2.69 \%$ & $3.0 \%$ \\
\hline 2413 & $3.14 \%$ & $1.34 \%$ & $4.0 \%$ \\
\hline 2431 & $3.62 \%$ & $4.03 \%$ & $3.0 \%$ \\
\hline 3124 & $3.86 \%$ & $4.30 \%$ & $7.0 \%$ \\
\hline 3142 & $4.35 \%$ & $3.50 \%$ & $7.0 \%$ \\
\hline 3214 & $3.86 \%$ & $6.99 \%$ & $1.0 \%$ \\
\hline 3241 & $3.62 \%$ & $3.76 \%$ & $3.0 \%$ \\
\hline 3412 & $2.17 \%$ & $2.96 \%$ & $1.0 \%$ \\
\hline 3421 & $4.35 \%$ & $4.57 \%$ & $2.0 \%$ \\
\hline 4123 & $3.38 \%$ & $4.57 \%$ & $1.0 \%$ \\
\hline 4132 & $6.28 \%$ & $3.76 \%$ & $3.0 \%$ \\
\hline 4213 & $3.62 \%$ & $5.91 \%$ & $6.0 \%$ \\
\hline 4231 & $2.90 \%$ & $3.23 \%$ & \\
\hline 4312 & $4.35 \%$ & $5.65 \%$ & \\
\hline 4321 & $\mathbf{7 . 7 3 \%}$ & $\mathbf{1 2 . 1 0 \%}$ & \\
\hline
\end{tabular}

The numbers are given in descending order. For example 2314 means that player 2 (the bidder that moved second), made the highest bid. Player 3 made the second highest bid, etc.

***: Indicates subgame perfect ordering

Table 3b: Bidding Order Percentages, 3-Player Data, All Sessions

\begin{tabular}{|r|r|r|}
\hline Bidding Order & $\begin{array}{c}\text { Percentage of Periods } \\
\text { Sequence is } \\
\text { Observed: PN3, All } \\
\text { Periods }\end{array}$ & $\begin{array}{c}\text { Percentage of Periods } \\
\text { Sequence is Observed: } \\
\text { PN3, Last 10 periods }\end{array}$ \\
\hline $123^{\star * *}$ & $29 \%$ & $40 \%$ \\
\hline 132 & $17 \%$ & $30 \%$ \\
\hline 213 & $25 \%$ & $30 \%$ \\
\hline 231 & $4 \%$ & $0 \%$ \\
\hline 312 & $11 \%$ & $0 \%$ \\
\hline 321 & $14 \%$ & $0 \%$ \\
\hline
\end{tabular}


Table 4: Estimates of Model of Cutoff Behavior

\begin{tabular}{|l|l|l|l|l|l|l|l|}
\hline $\begin{array}{l}\text { Equation and } \\
\text { data used }\end{array}$ & Constant & Period & Anonymous & $\begin{array}{l}\text { Cutoff } \\
\text { By J }\end{array}$ & $\begin{array}{l}\text { Cutoff By } \\
\text { Others }\end{array}$ & $\begin{array}{l}\text { Cutoffs } \\
\text { Observed }\end{array}$ & $\begin{array}{l}\text { Log- } \\
\text { Likelihood }\end{array}$ \\
\hline $\begin{array}{l}\text { 4 Players with } \\
\text { resale }\end{array}$ & $-.5278^{*}$ & $-.0572^{*}$ & .1957 & -.0460 & -.0208 & $.1169^{*}$ & -244.51 \\
$(.2572)$ & $(.0169)$ & $(.2220)$ & $-.0599)$ & $(.0366)$ & $(.0192)$ & $(400$ obs. $)$ \\
\hline $\begin{array}{l}\text { 4 Players } \\
\text { without resale }\end{array}$ & -.4457 & .0942 & ----- & -.1896 & -.1011 & -.0514 & -44.951 \\
$(.4786)$ & $(.0736)$ & & $(.2081)$ & $(.1831)$ & $(.0800)$ & $(68$ obs. $)$ \\
\hline $\begin{array}{l}3 \text { Players } \\
\text { without resale }\end{array}$ & $-1.999^{*}$ & .0546 & ----- & .2418 & -.6348 & .1034 & -26.116 \\
$(.7255)$ & $(.0653)$ & & $(.2165)$ & $(.3722)$ & $(.1530)$ & $(53$ obs. $)$ \\
\hline
\end{tabular}

* indicates significant at the 5\% level 